\title{
Fundamental M-dwarf parameters from high-resolution spectra using PHOENIX ACES models *
}

\section{Parameter accuracy and benchmark stars}

\author{
V. M. Passegger, S. Wende-von Berg, and A. Reiners
}

\begin{abstract}
Institut für Astrophysik, Georg-August-Universität Göttingen, Friedrich-Hund-Platz 1, 37077 Göttigen, Germany e-mail: vmpasseg@astro.physik.uni-goettingen.de
\end{abstract}

Received 10 July 2015 / Accepted 15 December 2015

\begin{abstract}
M-dwarf stars are the most numerous stars in the Universe; they span a wide range in mass and are in the focus of ongoing and planned exoplanet surveys. To investigate and understand their physical nature, detailed spectral information and accurate stellar models are needed. We use a new synthetic atmosphere model generation and compare model spectra to observations. To test the model accuracy, we compared the models to four benchmark stars with atmospheric parameters for which independent information from interferometric radius measurements is available. We used $\chi^{2}$-based methods to determine parameters from high-resolution spectroscopic observations. Our synthetic spectra are based on the new PHOENIX grid that uses the ACES description for the equation of state. This is a model generation expected to be especially suitable for the low-temperature atmospheres. We identified suitable spectral tracers of atmospheric parameters and determined the uncertainties in $T_{\text {eff }}, \log g$, and $[\mathrm{Fe} / \mathrm{H}]$ resulting from degeneracies between parameters and from shortcomings of the model atmospheres. The inherent uncertainties we find are $\sigma_{T_{\text {eff }}}=35 \mathrm{~K}, \sigma_{\log g}=0.14$, and $\sigma_{[\mathrm{Fe} / \mathrm{H}]}=0.11$. The new model spectra achieve a reliable match to our observed data; our results for $T_{\text {eff }}$ and $\log g$ are consistent with literature values to within $1 \sigma$. However, metallicities reported from earlier photometric and spectroscopic calibrations in some cases disagree with our results by more than $3 \sigma$. A possible explanation are systematic errors in earlier metallicity determinations that were based on insufficient descriptions of the cool atmospheres. At this point, however, we cannot definitely identify the reason for this discrepancy, but our analysis indicates that there is a large uncertainty in the accuracy of M-dwarf parameter estimates.
\end{abstract}

Key words. stars: low-mass - line: formation - stars: atmospheres - brown dwarfs - line: profiles - techniques: spectroscopic

\section{Introduction}

Determining atmospheric parameters in M dwarfs is very different from the situation in Sun-like stars. The complexity of cool atmospheres is dramatically enhanced because the main opacity sources are molecules and not atoms. The formation and abundance of these molecules is a complex process, and molecular absorption bands consist of thousands of individual lines that are not at all or sometimes only poorly known. For example, Fischer \& Valenti (2005) determined effective temperature, surface gravity, and metallicity in a sample of $1040 \mathrm{~F}-$-, G-, and $\mathrm{K}$-type stars. The precision they achieved in terms of $1 \sigma$ uncertainties is $44 \mathrm{~K}$ for $T_{\text {eff }}, 0.06$ dex for $\log g$, and 0.03 dex for abundances. Accurate determinations of low-mass star atmospheric parameters, on the other hand, still do not provide a clear picture, in particular when metallicity is concerned.

Previous work trying to determine stellar properties for low-mass stars generally dealt with each parameter separately. Rojas-Ayala et al. (2012) investigated near-infrared K-band spectra of $133 \mathrm{M}$ dwarfs. They determined effective temperatures by using the $\mathrm{H} 2 \mathrm{O}-\mathrm{K} 2$ index that quantifies absorption due to $\mathrm{H} 2 \mathrm{O}$ opacity. For calibration they used BT-Settl models (Allard et al. 2011) of solar metallicity. The uncertainties in temperature lie below $100 \mathrm{~K}$. Another approach was used by Boyajian et al. (2012), who calculated the effective temperature for nearby $\mathrm{K}$ and $\mathrm{M}$ dwarfs through interferometrically

* Based on observations carried out with UVES at ESO VLT. determined radii and bolometric fluxes from photometry. All uncertainties lie below $80 \mathrm{~K}$. The same approach was used by van Belle \& von Braun (2009). They calculated effective temperatures for a wide range of spectral types, including two mainsequence M dwarfs. Gaidos \& Mann (2014) used PHOENIX model atmospheres (Hauschildt et al. 1997; Allard et al. 2001) to determine the effective temperature from spectra observed in the visible wavelength range. For spectra taken in near-infrared $\mathrm{K}$ band they used spectral curvature indices. Different methods have been used to determine the surface gravity. Ségransan et al. (2003) used interferometry to determine the angular diameter of the stars: together with mass-luminosity relations, the mass can be derived and the surface gravity can be easily calculated. Other authors, for example, Rice et al. (2015) and del Burgo et al. (2013), used model fits to directly determine the surface gravity, which avoids assumptions about radius, mass, and age. Determining the metallicity in $\mathbf{M}$ dwarfs is more difficult than determining temperature and surface gravity. After several decades of research, different methods and models can still give contradicting results, hence we decided to discuss this topic in more detail below.

The measurement of metallicities in $\mathrm{M}$ dwarfs is different to the determination in Sun-like stars because the dense forest of spectral features complicates a line-by-line approach. Instead, a full spectral synthesis of the considered spectral range is preferable, which is generally much more complex than computing individual lines. Early attempts to measure metallicities 
for M dwarfs date back to Mould (1976), who performed a line-by-line analysis of atomic near-IR lines. Jones et al. (1996) used PHOENIX model spectra (Hauschildt et al. 1999; Allard et al. 2001) to follow a similar approach, and Gizis (1997) matched low-resolution optical spectra to the same models. One of the first analyses of a high-resolution $\mathrm{M}$ dwarf spectrum was performed by Valenti et al. (1998) and Zboril \& Byrne (1998), trying to fit high-resolution spectra to PHOENIX models. They concluded that their M-dwarf metallicities were only indicative, a conclusion that is probably valid for all previous references. As an example for the problems inherent to M-dwarf metallicity determinations, we can compare the values for one star found with different analyses; for the mid-M object Gl 725B (M 3.5), Valenti et al. (1998) reported $[\mathrm{Fe} / \mathrm{H}]=-0.92$, while Zboril \& Byrne (1998) found $[\mathrm{Fe} / \mathrm{H}]=-0.15$. The results for the probably best-known mid-M dwarf, Barnard's star (GJ 699, M4), which we also investigated here, are spread in the literature between $[\mathrm{Fe} / \mathrm{H}]=-0.39 \pm 0.17$ from Rojas-Ayala et al. (2012) and -0.75 from Jones et al. (1996). Rojas-Ayala et al. (2012) analysed the equivalent widths of $\mathrm{NaI}$ and $\mathrm{CaI}$ and calibrated their scale using metallicities of $18 \mathrm{FGK}+\mathrm{M}$ binary systems.

An important indication that metallicity severely affects the energy distribution in low-mass stars was provided by Delfosse et al. (2000). The authors used direct mass determinations from measurements in binary systems to derive an empirical massluminosity relation. They found that while this relation shows relatively little scatter in the $K$ band, the scatter in the $V$ is huge. More specifically, the colour index $V-K$ shows large scatter as a function of mass, which can be explained by a scatter in metallicities, as was shown using predictions of $V-K$ colours calculated from the PHOENIX models. Clearly, metallicity plays a crucial role for the spectra at visual wavelengths, while it is not as important at near-IR wavelengths.

A quantitative improvement to metallicity calculations in M dwarfs was achieved when Ségransan et al. (2003) reported interferometric radius measurements of $\mathrm{M}$ dwarfs. Direct radius measurements together with the luminosity of and the distance to a star provide independent constraints to effective temperature by removing one free parameter. Furthermore, the massluminosity relation from Ségransan et al. (2003) can estimate the mass as a function of the near-IR luminosity reasonably well, so that together with the radius, the surface gravity is known as well. In systems with direct radius measurements, measurements of the apparent luminosity, and accurate determinations of the distance, metallicity remains the only free parameter in the description of a stellar atmosphere. This concept was applied to two nearby M dwarfs by Woolf \& Wallerstein (2004) and Dawson \& De Robertis (2004) using NEXTGEN model atmospheres (Hauschildt et al. 1999). Woolf \& Wallerstein (2005) measured metallicities in $15 \mathrm{M}$ dwarfs for which no direct radius measurements were available. Bonfils et al. (2005) enriched this sample with 20 systems consisting of an F-, G-, or K-dwarf primary and an M-dwarf secondary. These binaries are believed to have formed together so that their metal abundance is expected to be identical, which provides the only independent test of M-star metallicity. A similar approach was chosen by Bean et al. (2006b,a), who used five binaries to determine the metal content of the primary and from this deduced the metal content of the M-dwarf secondaries. The model they developed was then used to determine the metallicity of three planet-hosting M dwarfs. Bonfils et al. (2005) also provided a colour-metallicity relation, from which the metal abundance of $M$ dwarfs can apparently be derived simply from their colours. This reduces the complex problem of atmospheric physics in $\mathrm{M}$ dwarfs to a two-dimensional relation between infrared and visual luminosities and has been used in many following studies since, for example by Casagrande et al. (2008) and for the calibration applied in Neves et al. (2013). However, Rojas-Ayala et al. (2012) found that the results of Bonfils et al. (2005) show lower metallicity values for stars with solar and super-solar metallicities. Gaidos \& Mann (2014) used empirical relations between metallicity and atomic line strength in the $H$ and $K$ band calibrated with FGK+M binary systems as described by Mann et al. (2013). Their results in metallicity agree excellently well with those of Rojas-Ayala et al. (2012), with mean differences of 0.03 dex. A recent work by Maldonado et al. (2015) used pseudo-equivalent widths of optical spectra to determine effective temperature and $[\mathrm{Fe} / \mathrm{H}]$. They showed that the calibration of Casagrande et al. (2008) underestimates temperatures because Casagrande et al. (2008) assumed M dwarfs to be blackbodies, but M dwarfs have more flux in the infrared than predicted for a blackbody. For metallicities, however, Maldonado et al. (2015) reported good agreement of their results with Neves et al. (2012) and Neves et al. (2014).

A surprising result that arose from the comparison between metallicities in Sun-like planet-hosting stars and M-dwarf planet-hosting stars was made by Bean et al. (2006b), who claimed that Sun-like planet hosts tend to have high metal abundances, but this is not the case for M-dwarf planet hosts. This result was disproved by several subsequent works (e.g. Johnson \& Apps 2009; Rojas-Ayala et al. 2012; Gaidos \& Mann 2014), which showed that M-dwarf planet hosts also have solar to super-solar metallicities. One way to explain the discrepancy found by Bean et al. (2006b) is that metallicity determinations for M dwarfs are systematically underestimating the metal abundance, implying that the colour-metallicity relations are not generally applicable either. This alternative was tested by Johnson \& Apps (2009), who compared metallicity measurements in Sunlike stars and $\mathrm{M}$ dwarfs in the overlapping region where different calibration methods are valid. They showed that the metallicities of the high-metal $\mathrm{M}$ dwarfs in their sample are underestimated by as much as $0.32 \mathrm{dex}$ in $[\mathrm{Fe} / \mathrm{H}]$ on average. Neves et al. (2012) reported that the method of Johnson \& Apps (2009) is a good predictor for high metallicities, but tends to overestimate low-metallicity M dwarfs by about 0.14 dex. Rojas-Ayala et al. (2012) came to the same conclusion. Schlaufman \& Laughlin (2010) inferred a photometric metallicity calibration and also found that Johnson \& Apps (2009) overestimated metallicities. Rojas-Ayala et al. (2012) reported that Schlaufman \& Laughlin (2010) underestimated values for some solar and super-solar metallicity stars, but for stars with sub-solar metallicity they either under- or overestimate by up to 0.6 dex. A method similar to the one we describe in this work was used by Rajpurohit et al. (2013). They compared low- and medium-resolution spectra of $152 \mathrm{M}$ dwarfs to BT-Settl models and applied a $\chi^{2}$-method to determine effective temperatures. Rajpurohit et al. (2014) improved this work by additionally determining $\log g$ and metallicity and interpolating between the model grid points for these two parameters. This was done for high-resolution spectra of $21 \mathrm{M}$ dwarfs. A comparison of available metallicity determinations was carried out by Neves et al. (2012). In their recent work, Mann et al. (2015) presented their temperature determination using optical spectra and BT-Settl models following the approach of Mann et al. (2014). For metallicity they used equivalent widths of atomic features in near-infrared spectra together with the empirical relation from Mann et al. (2013) and Mann et al. (2014). They found only slight differences to the metallicities reported by Rojas-Ayala et al. (2012), Neves et al. (2013), 
Table 1. Parameter space of the spectral grid.

\begin{tabular}{ccc}
\hline \hline & Range & Step size \\
\hline$T_{\text {eff }}[\mathrm{K}]$ & $2300-5900$ & 100 \\
$\log (g)$ & $0.0-+6.0$ & 0.5 \\
{$[\mathrm{Fe} / \mathrm{H}]$} & $-4.0--2.0$ & 1.0 \\
& $-2.0-+1.0$ & 0.5 \\
\hline
\end{tabular}

and Neves et al. (2014). In this paper we aim for a fresh attempt to determine the atmospheric parameters of cool stars. We take advantage of the new PHOENIX model grid (Husser et al. 2013), which makes use of significantly advanced microphysics relevant to M-dwarf atmospheres. In Sect. 2 we describe the new model, and in Sect. 3 we introduce our method to derive atmospheric properties from comparison between synthetic spectra and observations. Observational data from benchmark stars are described in Sect. 4, and an analysis of these stars is carried out in Sect. 5, before we summarise our results in Sect. 6.

\section{PHOENIX ACES model atmospheres}

For the spectral fitting we used the latest PHOENIX grid ACES (see Husser et al. 2013) ${ }^{1}$. It makes use of a new equation of state, which accounts especially for the formation of molecules at low temperatures. Hence it is ideally suited for the synthetisation of cool stars spectra. The 1D models are computed in planeparallel geometry and consist of 64 layers. Convection is treated in mixing-length geometry, and from the convective velocity a microturbulent velocity is deduced via $v_{\text {mic }}=0.5 \cdot v_{\text {conv }}$ ( see Wende et al. 2009). Both are used to compute the high-resolution spectra. An overview of the model grid parameters is shown in Table 1 . In all models the assumption of local thermal equilibrium is used.

First comparisons of these models with observations show that the quality of the computed spectra is greatly improved in comparison to older versions. Especially the regions of the TiO bands in the optical are now well fitted. These regions are some of the key regions to determine effective temperatures (see below). One problem in previous PHOENIX versions was that the $\epsilon$ - and $\gamma$-TiO bands in observations could not be reproduced with the same effective temperature (Reiners 2005). This problem is now solved in the current version of the code (see below).

\section{Parameter determination method}

Since the parameters, $T_{\text {eff }}, \log g$, and metallicity in M-type stars can be strongly degenerate, it is necessary to use spectral regions that are sensitive to one or more stellar parameters simultaneously to break the degeneracies. To determine the best set of parameters for a given star, a $\chi^{2}$-method is appropriate. The details of the method used here are described in the following.

\subsection{Spectral regions}

We chose several spectral regions that exhibit different dependencies on the three stellar parameters. We chose the molecular TiO bands around 7050 and $8430 \AA$ ( $\gamma$ - and $\epsilon$ - electronic transitions, respectively) since these oxide bands are very sensitive to effective temperature, but almost insensitive to surface gravity

\footnotetext{
1 http://phoenix . astro.physik. uni-goettingen.de/
}
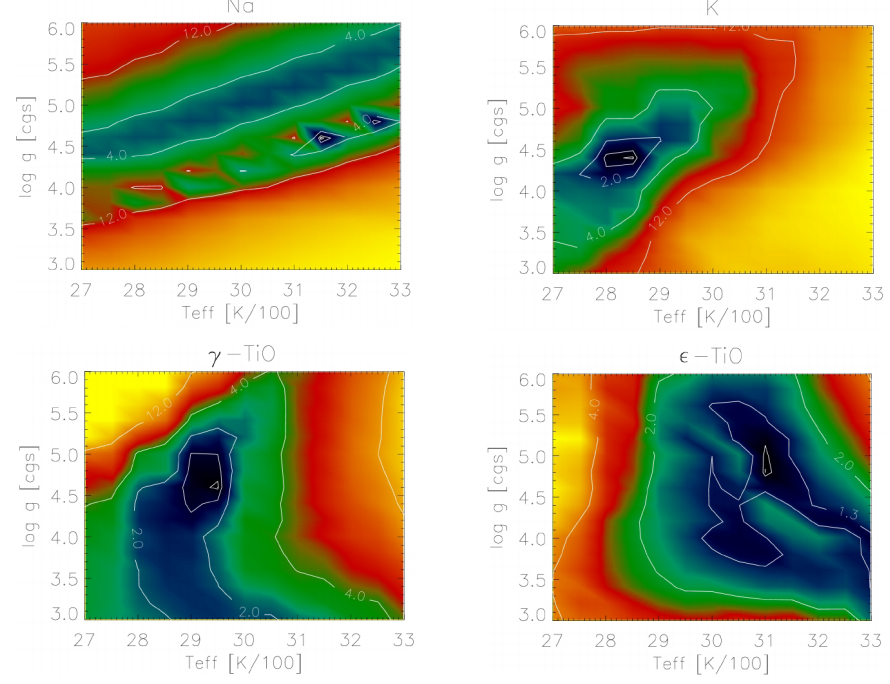

Fig. 1. $\chi^{2}$-maps of the used oxygen bands and alkali lines for the $\mathrm{M}$ dwarf GJ551 with $\log g=5.02, T_{\text {eff }}=2927 \mathrm{~K},[\mathrm{Fe} / \mathrm{H}]=-0.07$, and $S / N \sim 100$. We show the fit quality in the $T_{\text {eff }}-\log g$ plane after calculating a rough global minimum on the low-resolution grid.
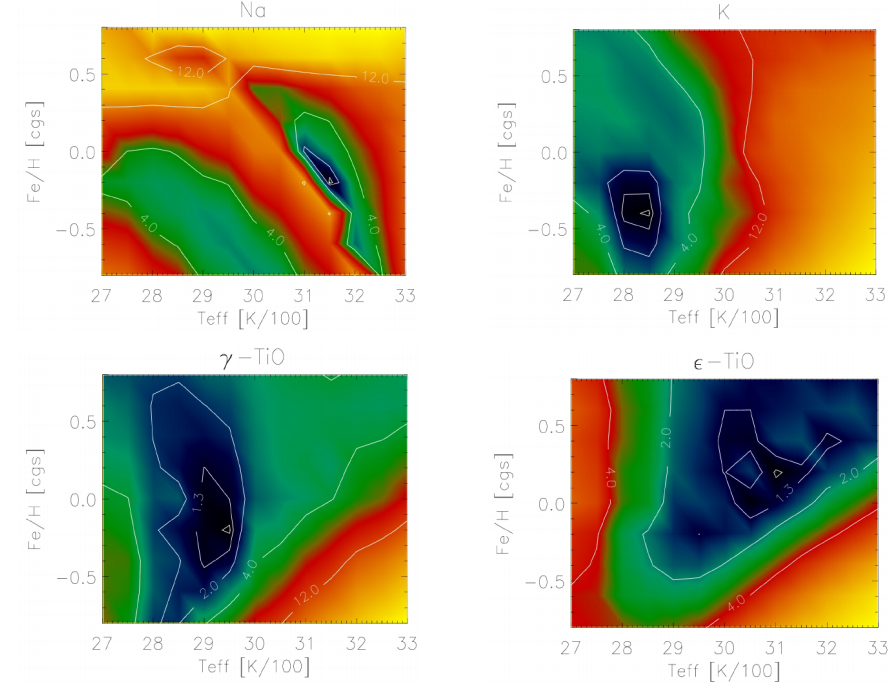

Fig. 2. $\chi^{2}$-maps of the used oxygen bands and alkali lines for the $\mathrm{M}$ dwarf GJ551 with $\log g=5.02, T_{\text {eff }}=2927 \mathrm{~K},[\mathrm{Fe} / \mathrm{H}]=-0.07$, and $S / N \sim 100$. We show the fit quality in the $T_{\text {eff }}-[\mathrm{Fe} / \mathrm{H}]$ plane after calculating a rough global minimum on the low-resolution grid.

(Fig. 1). For cooler stars, as shown here, a dependence on surface gravity is introduced by the increasing micro-turbulent velocities, which enhance the line width towards lower $\log g$ values (see Wende et al. (2009) for $\mathrm{FeH}$ as an example). More sensitive to surface gravity are alkali lines (Fig.1), which show large alterations of their line wings that are due to pressure broadening (in case of the $\mathrm{K}$ lines it is more pronounced towards cooler temperatures). We chose the K- and Na-line pairs at around $7680 \AA$ and $8190 \AA$, respectively. To determine the metallicity, we can use all these regions as well because the $\epsilon$-TiO band and the alkali lines are strongly dependent on this quantity (see Fig. 2). Rajpurohit et al. (2014) also used the K- and Na-line pairs because of their sensitivity to gravity and metallicity, as well as several TiO-bands for temperature determination. They also 

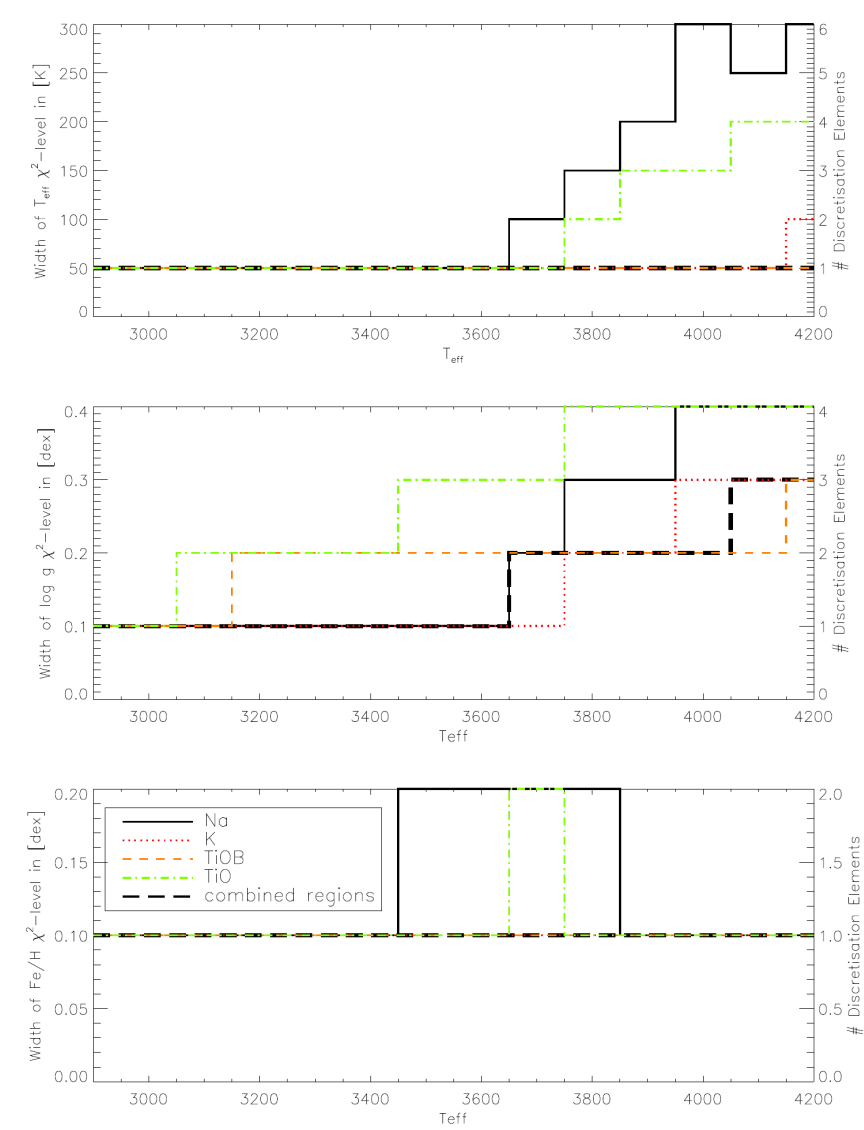

Fig. 3. Changes in sensitivity on certain parameters for different lines with increasing temperature. The ciel-function is used because only discrete numbers can be resolved.

illustrated the dependency on the different stellar parameters. The chosen spectral regions are also affected by lines of the Earth's atmosphere. Especially the K- and Na-line pairs are contaminated by $\mathrm{O} 2$ and $\mathrm{H} 2 \mathrm{O}$ bands, respectively. We used masks to exclude the atmospheric lines from the fit. The contamination of the TiO bands around 7050 and $8430 \AA$ is very weak and can be neglected. The degree of sensitivity on a certain parameter for a particular line (band) also varies. In Fig. 3 we show how the width of a $\chi^{2}$-level in a $\chi^{2}$-map changes with varying effective temperature (i.e. the $T_{\text {eff }}$ of the synthetic spectrum from which the $\chi^{2}$-map is produced is varied) for a fixed surface gravity of $\log g=5.0 \mathrm{cgs}$ and metallicity of $z=0.0 \mathrm{cgs}$. The change is also measured in terms of discretion elements in the $\chi^{2}$-maps, since this determines the given resolution (left axis). All investigated regions become less gravity sensitive towards higher $T_{\text {eff }}$, which is probably due to the increasing thermal broadening, which becomes stronger than the pressure broadening (see also Sect. 3.3). At this point we have to keep in mind that the sensitivity curves only represent the behaviour for the chosen parameter values. They look different for other metallicities or surface gravities, which are not shown in detail here. Nevertheless, it becomes clear that the combined $\chi^{2}$-map of all regions is the best choice for most temperatures and provides the highest sensitivity. Even though the sensitivity of the $\mathrm{K}$ lines is better for most temperatures than the combined one, we would not suggest to use only these lines because the results would then only depend on two lines. Experience in fitting real observations showed that the results deduced from the $\mathrm{K}$ lines alone can differ from the combined solution where the latter matches the actual parameters to a higher accuracy.

\section{2. $\chi^{2}$-method}

To determine the stellar parameters, we basically used a $\chi^{2}$ reduction. We started with the $\chi^{2}$ determination using a lowresolution grid of atmospheres in a wide range around the expected parameters of the object of interest. In this step, the models were first convolved to match the observed spectral resolution. Then we normalised the average flux of the models and the observed spectra to unity. After this, the models were interpolated to the wavelength grid of the observed spectra. Finally, every model of the grid was compared to the observed spectrum at each wavelength point, and the $\chi^{2}$ was calculated to determine a rough global minimum. To obtain more pronounced minima in the $\chi^{2}$-map, a dynamical mask was applied to every model spectrum. Figures 1 and 2 show the $\chi^{2}$-maps after this first step for the different wavelength ranges we used. In the next step we used the IDL curvefit-function to determine accurate stellar parameters around a narrow range of the minimum using linear interpolation inside the model grid. To do this, we used the three smallest local minima from the combined $\chi^{2}$-map as start values for the fitting algorithm to search for a global minimum that may be located between the grid points of the $\chi^{2}$-map.

\subsubsection{Dynamical mask and re-normalisation}

As already mentioned, not all parts of the spectrum show the same dependence on the stellar parameter. In certain parameter regions, certain lines react stronger or weaker to variations. To weight the very sensitive lines more in the $\chi^{2}$-determination, we applied a dynamical mask that depends on the model spectrum currently applied to the observed spectrum. In a first step, we produced a synthetic mean spectrum, constructed from all model spectra within the parameter range used for $\chi^{2}$ calculation. Then the difference between the mean spectrum and the model spectrum, which should be applied to the observations, was determined by dividing the model by the mean spectrum. Those regions showing a wide difference (i.e. ratios very different from 1) indicate wavelength regions that are very sensitive to the actual parameter configuration. These regions were masked and used as weighting in the $\chi^{2}$ calculation. Applying such a mask has no basic influence on finding the best fit, but results in more pronounced minima in the $\chi^{2}$-maps. To account for slight differences of the continuum level and possible linear trends between the already normalised observed and computed spectra, we applied a re-normalisation. We used

$F_{\text {re-norm }}^{\text {obs }}=F^{\text {obs }} \cdot \frac{\text { continuum fit }}{\text { model }}$ continuum fit observation $_{\text {, }}$

where the continuum fits are linear. This has no significant influence on the positions of the minima, but improves the overall accuracy.

\subsubsection{Fit algorithm}

The IDL curvefit-function turned out to be the most stable, accurate, and fastest algorithm to determine the set of parameters. We also tested the IDL powell- and amoeba-algorithms. Since the curvefit-function requires continuous parameters, we performed a three-dimensional linear interpolation in the computed spectra on the grid shown in Table 1. The interpolation was made for each wavelength point in the required wavelength range. 
V. M. Passegger et al.: Fundamental M-dwarf parameters from high-resolution spectra using PHOENIX ACES models. I.
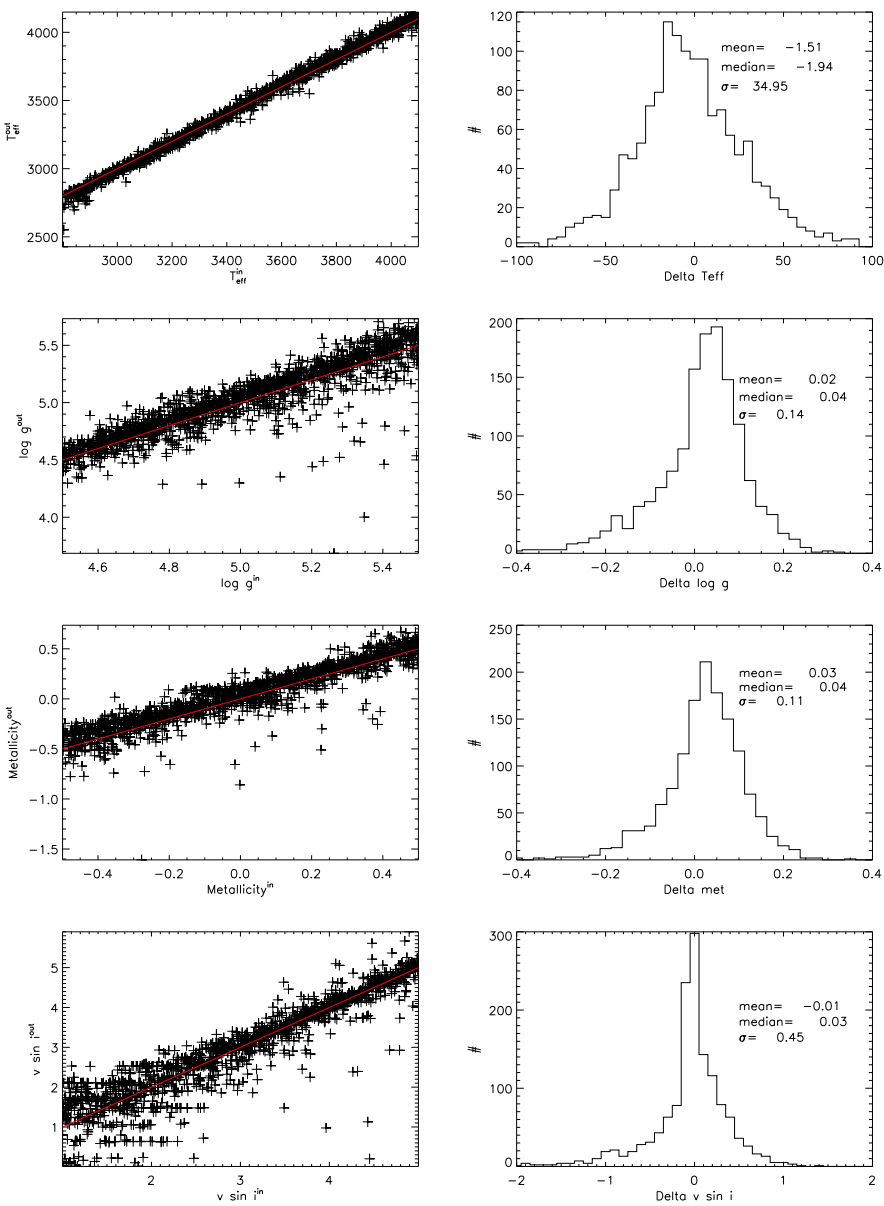

Fig. 4. Comparison and deviations between output and input parameters. Left columns: input (not starting) parameters against determined parameters. The diagonal line represents a one-to-one correspondence. Right columns: histograms of the deviations between output and input parameters.

\subsection{Precision of the method}

To test the precision of our method, we produced a set of 1400 spectra with uniformly distributed random parameters $\left(T_{\mathrm{eff}}, \log g,[\mathrm{Fe} / \mathrm{H}]\right.$, and $v \sin i$ ) and different resolutions of $R=100000,44000$, and 10000 . We added Poisson noise to simulate a signal-to-noise ratio $(\mathrm{S} / \mathrm{N})$ of $\sim 100$. The starting values differ from the actual parameters in a range of $\pm 50 \mathrm{~K}$ for $T_{\text {eff }}, \pm 0.25 \mathrm{dex}$ for $\log g$ and $[\mathrm{Fe} / \mathrm{H}]$, and $\pm 1 \mathrm{kms}^{-1}$ for rotational broadening. We also used a continuum normalisation different to the one used in the fitting routine to simulate normalisation effects. We used the method described above to determine the parameters and show the deviations from input to output parameters in the set of histograms in Fig. 4 for $R=100000$.

The deviation from a mean residual value of zero probably stems from the fitting algorithm, since we observe a change in sign between the curvefit-function and the amoeba-algorithm. The two algorithms use different convergence criteria, which we believe results in the offset. In the presented case all values except for the resolving power (or $v \sin i$ ) are positive, which means that the determined parameters are slightly higher than the actual ones.

We find standard deviations from the mean value of $35 \mathrm{~K}$ for $T_{\mathrm{eff}}, 0.14 \mathrm{dex}$ for $\log g, 0.11 \mathrm{dex}$ for $[\mathrm{Fe} / \mathrm{H}]$, and $0.5 \mathrm{kms}^{-1}$ for $v \sin i$. No Gaussian FWHM was used since the residual distributions are not symmetric. The reason for the asymmetry in the $\log g$ and $[\mathrm{Fe} / \mathrm{H}]$ plot is probably due to the logarithmic character of the quantity. For low effective temperatures the derived temperatures are systematically lower. This changes towards high temperatures into the opposite. This is likely related to the temperature-sensitive $\mathrm{TiO}$ bands, which are hardly present for high temperatures and start to saturate towards the cooler end. However, the average deviations at both ends are about $50 \mathrm{~K}$ and agree with the final precision.

In the plot for the rotational velocities, the largest scatter is found for values below $3 \mathrm{~km}$. This is explained by the instrumental resolution that we set to $R=100000$, which equals a broadening of $\sim 3 \mathrm{~km}$. Hence lower velocities cannot be expected to be resolved.

For lower resolutions the standard deviations will increase because the spectra contain less information and close lines begin to overlap. For resolutions around $R=44000$ (like our observed spectra from GJ441 and GJ551, see next section) the deviations are $40 \mathrm{~K}$ for $T_{\text {eff }}, 0.18$ dex for $\log g, 0.14$ dex for $[\mathrm{Fe} / \mathrm{H}]$, and $1 \mathrm{kms}^{-1}$ for $v \sin i$. For $R=10000$ the deviations are $124 \mathrm{~K}$ for $T_{\text {eff }}, 0.46$ dex for $\log g, 0.36$ dex for $[\mathrm{Fe} / \mathrm{H}]$, and $7.5 \mathrm{kms}^{-1}$ for $v \sin i$. However, because of the resolution we do not expect to resolve rotational velocities lower than $\sim 7 \mathrm{kms}^{-1}$ for $R=44000$ and $\sim 30 \mathrm{kms}^{-1}$ for $R=10000$.

\section{Observational data}

\subsection{Observed data}

The observational data used to determine the spectral parameters are UVES spectra. The spectrum of GJ411 was taken under the program ID 74.B-0639 with a resolution of $R \sim 46000$. GJ551 was observed under the program ID 73.C-0138 with a resolving power of $R \sim 42300$. The spectra of GJ667C and GJ699 were taken under the program ID 87.D-0069. The highresolution mode with a slit width of 0.3 " was used, leading to a resolving power of $R \sim 100000$. The observations cover a wavelength range from $640 \mathrm{~nm}$ to $1020 \mathrm{~nm}$ on the two red chips. For GJ411 and GJ551 the ESO PHASE3 data products were used. The data for GJ667C and GJ699 were reduced using the ESOREX pipeline for UVES. The wavelength solution is based on the Th-Ar calibration frames. All orders were corrected for the blaze function and also normalised to unity continuum level. Then all orders were merged. For overlapping orders (at shorter wavelengths) the red sides were used because their quality is better. Some of our spectra contain orders merged into a onedimensional spectrum; this merging of orders can potentially lead to artefacts caused by incorrect order merging, such as line deformation or alteration of line depths, which is particularly problematic if spectral features used to determine the parameter are influenced. Our experience is that the ESO pipelines perform order merging very carefully with little systematic influence. Moreover, we used a number of different features in our fitting procedure that are unlikely to systematically fall into regions close to order edges. We therefore believe that potential defects from individual lines and order merging are negligible. In a last step we removed bad pixels and cosmics.

\subsection{Objects}

We wish to test the results determined from our PHOENIX models in stars with well-studied parameters. We used four objects for these benchmark tests. The first three are stars for which interferometric radius determinations (Ségransan et al. 2003) 
allow an independent estimate of their surface gravity and temperature together with information on luminosity and distance (Table 3). Our fourth benchmark object, GJ 667C, was used for a comparison of metallicity that is known for its binary companions. This fourth object mainly serves as an additional consistency check, a more systematic investigation considering a larger sample of binary components will be carried out in a subsequent paper when a larger sample of M-dwarf spectra is analysed. All four benchmark stars are very slow rotators with no indication for rotational line broadening. The first three objects also have measured rotation periods consistent with very slow rotation and low activity (see Reiners et al. 2012).

- GJ411 (M2): this is the hottest star we investigated. With its slow rotation and no signs of high magnetic activity, it is an ideal target for testing the new model atmospheres at the warm end of M-dwarf temperatures. Radius measurements and a determination of $\log g$ are taken from Ségransan et al. (2003). Temperature estimates derived from interferometric radius and luminosity are $T_{\text {eff }}=3593 \pm 60 \mathrm{~K}$ (van Belle \& von Braun 2009) and $T_{\text {eff }}=3460 \pm 37 \mathrm{~K}$ (Boyajian et al. 2012). Results from spectroscopy yielded temperature estimates of $T_{\text {eff }}=3526 \pm 18 \mathrm{~K}$ (Rojas-Ayala et al. 2012), $T_{\text {eff }}=3697 \pm 110 \mathrm{~K}$ (Gaidos \& Mann 2014) and $T_{\text {eff }}=3563 \pm 60 \mathrm{~K}$ (Mann et al. 2015). The metallicity of this star was reported as $[\mathrm{Fe} / \mathrm{H}]=-0.4$ (Woolf $\&$ Wallerstein 2005), $[\mathrm{Fe} / \mathrm{H}]=-0.41 \pm 0.17$ (Rojas-Ayala et al. 2012), $[\mathrm{Fe} / \mathrm{H}]=-0.35 \pm 0.08$ (Neves et al. 2013), $[\mathrm{Fe} / \mathrm{H}]=-0.3 \pm 0.08$ (Gaidos \& Mann 2014), and most recently $[\mathrm{Fe} / \mathrm{H}]=-0.38 \pm 0.08$ by Mann et al. (2015).

- GJ699 (Barnard's star, M4): barnard's star is of the most frequently observed and well-studied stars. It is classified as an M4 dwarf and shows no indications for enhanced magnetic activity in the spectra. Radii measurements are reported in Ségransan et al. (2003) together with $\log g$. Boyajian et al. (2012) derived a temperature of $T_{\text {eff }}=3230 \pm 10 \mathrm{~K}$ from radius and luminosity data. Spectroscopic temperatures reported are $T_{\text {eff }}=3266 \pm 29 \mathrm{~K}$ (Rojas-Ayala et al. 2012), $T_{\text {eff }}=3338 \pm 110 \mathrm{~K}$ (Neves et al. 2014), $T_{\text {eff }}=3247 \pm$ $61 \mathrm{~K}$ (Gaidos \& Mann 2014), and $T_{\text {eff }}=3228 \pm 60 \mathrm{~K}$ (Mann et al. 2015). Metallicities reported for this star are spread between $[\mathrm{Fe} / \mathrm{H}]=-0.39$ and -0.75 , recent examples are $[\mathrm{Fe} / \mathrm{H}]=-0.39 \pm 0.17$ (Rojas-Ayala et al. 2012), $[\mathrm{Fe} / \mathrm{H}]=-0.52 \pm 0.08$ (Neves et al. 2013), $[\mathrm{Fe} / \mathrm{H}]=-0.51 \pm$ 0.09 (Neves et al. 2014), $[\mathrm{Fe} / \mathrm{H}]=-0.32 \pm 0.08$ (Gaidos \& Mann 2014), and $[\mathrm{Fe} / \mathrm{H}]=-0.40 \pm 0.08$ (Mann et al. 2015).

- GJ551 (M6): this M6 star is the coolest object in our sample. Like the other stars, no indications of enhanced activity were found and the spectra are consistent with slow rotation. $T_{\text {eff }}$ and $\log g$ from Ségransan et al. (2003) are shown in Table 3. Neves et al. (2013) derived a temperature of $T_{\text {eff }}=2659 \mathrm{~K}$, which is significantly lower than the value from interferometry. Reports of metallicity include $[\mathrm{Fe} / \mathrm{H}]=0.19($ Edvardsson et al. 1993), $[\mathrm{Fe} / \mathrm{H}]=0.0 \pm 0.08$ (Neves et al. 2013), $[\mathrm{Fe} / \mathrm{H}]=0.16 \pm 0.20$ (Neves et al. 2014), and $[\mathrm{Fe} / \mathrm{H}]=-0.03 \pm 0.09$ (Maldonado et al. 2015).

- GJ667C (M1.5): this M1.5V dwarf also is a slow rotator without signs of strong magnetic activity; it shows no $\mathrm{H} \alpha$ emission, for example. The star hosts several lowmass planets. Atmospheric parameters were reported in Anglada-Escudé et al. (2013). The star has no interferometric radius constraints, hence we lack independent information on temperature and gravity. Because it is part of a multiple star system, we have constraints on metallicity
Table 2. Metallicities measured for GJ 667AB.

\begin{tabular}{lc}
\hline \hline Reference & {$[\mathrm{Fe} / \mathrm{H}]$} \\
\hline Perrin et al. (1988) & -0.59 \\
Marsakov \& Shevelev (1988) & -0.52 \\
Thevenin (1998) & -0.55 \\
Santos et al. (2005) & -0.43 \\
Taylor (2005) & -0.66 \\
\hline
\end{tabular}

assuming that the three components of the triple share similar metallicities. For the K3V component of the system, we found five independent metallicity determinations that we collect in Table 2. The mean and median values of the sample are $[\mathrm{Fe} / \mathrm{H}]=-0.55$ with a standard deviation of $\sigma[\mathrm{Fe} / \mathrm{H}]=0.08$. For the temperature, literature data are $T_{\text {eff }}=3350 \pm 50 \mathrm{~K}$ (Anglada-Escudé et al. 2013), $T_{\text {eff }}=3351$ (Neves et al. 2013), $T_{\text {eff }}=3445 \pm 110 \mathrm{~K}$ (Neves et al. 2014), and $T_{\text {eff }}=3472 \pm 61 \mathrm{~K}$ (Gaidos \& Mann 2014). Spectroscopic determination of metallicity for the C component are $[\mathrm{Fe} / \mathrm{H}]=-0.55 \pm 0.1$ (Anglada-Escudé et al. 2013), $[\mathrm{Fe} / \mathrm{H}]=-0.53 \pm 0.08$ (Neves et al. 2013), $[\mathrm{Fe} / \mathrm{H}]=-0.50 \pm$ 0.09 (Neves et al. 2014). Gaidos \& Mann (2014) reported a value of $[\mathrm{Fe} / \mathrm{H}]=-0.3 \pm 0.08$ from an empirical relation between metallicity and atomic line strength, described in Mann et al. (2013).

\section{Results}

Figure 5 shows the spectra of all four stars together with the bestfit models. The atmospheric parameters derived from the comparison between our observational data and the new PHOENIX models are summarised in Table 3 . We visualise our results in comparison to literature data for effective temperature in the top panel of Fig. $6, \log g$ in the middle panel, and metallicity in the bottom panel of Fig. 6 .

\subsection{Effective temperature and surface gravity}

Our results for temperature and surface gravity are consistent with most of the literature data within $1 \sigma$ uncertainties (see top panel in Fig. 6). For GJ 551, the temperature from Neves et al. (2013) is significantly cooler than our result and the one from Ségransan et al. (2003). This discrepancy may be caused by the $\mathrm{TiO}$ bands starting to saturate at cooler temperatures, as mentioned in Sect. 3.3. This consistency is crucial for our test of the accuracy of the new PHOENIX ACES model generation. It shows that with the new model set we are able to obtain physically meaningful parameters of very cool stars. Furthermore, the fact that the synthetic spectra match the TiO bands as well as atomic lines with one consistent parameter set (see Fig. 5) indicates that the microphysics used for the new PHOENIX atmosphere grid has improved significantly with respect to older model generations (Reiners 2005). For GJ 411 and GJ 699 our results perfectly agree with those of Mann et al. (2015) within $10 \mathrm{~K}$.

\subsection{Metallicity}

For the three benchmark stars used above, GJ 411, GJ 699, and GJ 551, direct radius measurements provide independent information on temperature and surface gravity. The third crucial 
V. M. Passegger et al.: Fundamental M-dwarf parameters from high-resolution spectra using PHOENIX ACES models. I.
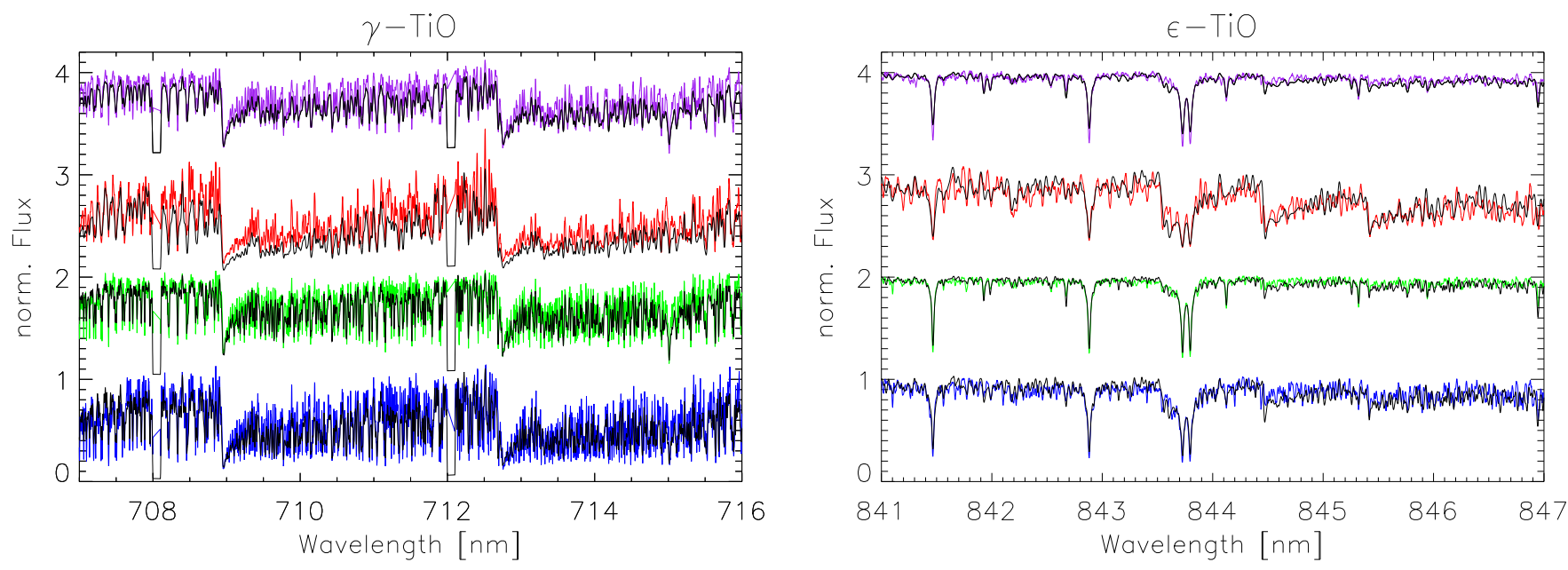

K
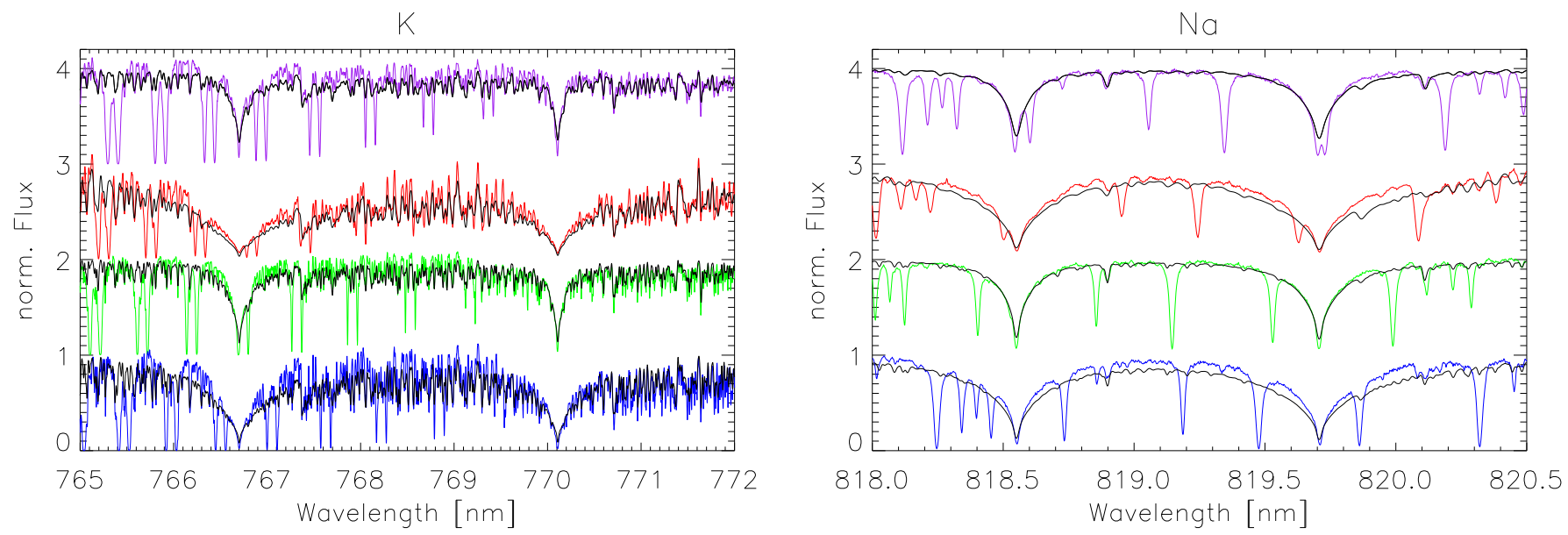

Fig. 5. Comparison of the new PHOENIX models (grey) to four stars with known temperature and surface gravity (colour; from top to bottom: GJ411, GJ551, GJ667C, and GJ699). The new models provide a substantial improvement over the old model generation (see Reiners 2005). In particular, the two TiO bands yield consistent results, although there might still be some problems fitting the K- and Na-lines (e.g. see spectrum of GJ551 and GJ699). The data were not corrected for absorption lines from Earth's atmosphere, therefore many sharp lines seen in the observations are not captured by the models. These lines were not included in the fit.

Table 3. Parameters for benchmark stars with interferometric radii.

\begin{tabular}{llllllll}
\hline \hline Object & $\mathrm{Sp}$ & $T_{\text {eff }}[\mathrm{K}]^{b}$ & $\log g^{b}$ & {$[\mathrm{Fe} / \mathrm{H}]^{a}$} & $T_{\mathrm{eff}}^{\mathrm{fit}}$ & $\log g^{\text {fit }}$ & {$[\mathrm{Fe} / \mathrm{H}]^{\mathrm{fit}}$} \\
\hline GJ411 & M2.0 & $3570 \pm 42$ & $4.85 \pm 0.03$ & $-0.35 \pm 0.08$ & $3565 \pm 40$ & $4.84 \pm 0.18$ & $+0.00 \pm 0.14$ \\
GJ699 & M4.0 & $3163 \pm 65$ & $5.05 \pm 0.09$ & $-0.52 \pm 0.08$ & $3218 \pm 35$ & $5.25 \pm 0.14$ & $-0.13 \pm 0.11$ \\
GJ551 & M5.5 & $3042 \pm 117$ & $5.20 \pm 0.23$ & $+0.00 \pm 0.08$ & $2927 \pm 40$ & $5.02 \pm 0.18$ & $-0.07 \pm 0.14$ \\
\hline
\end{tabular}

Notes. Columns 3-5: literature values; Cols. 6-9: parameters determined from our model fit. ${ }^{(a)}$ Neves et al. (2013). ${ }^{(b)}$ Ségransan et al. (2003).

atmospheric parameter, metallicity, is not independently constrained, but can only be determined from spectroscopic analysis. Literature values given in Table 3 are also based on spectroscopic models, which are often calibrated using components of binary stars, as discussed in the introduction. For the three stars GJ 411, GJ 699, and GJ 551, our metallicities are not fully consistent with the literature values of Neves et al. (2013); on average, our results are $\Delta[\mathrm{Fe} / \mathrm{H}]=0.22$ dex higher. As an independent test of our metallicity results, we consider the M1.5 dwarf GJ 667C, which is part of a triple system and often used to anchor M-dwarf metallicity scales. For the brighter components, $[\mathrm{Fe} / \mathrm{H}]$ is very accurately determined with a mean literature value of $[\mathrm{Fe} / \mathrm{H}]=-0.55$ (Sect. 4.2). This low-metallicity star should be a good indicator for whether our models can determine metallicities in $\mathrm{M}$ dwarfs with sub-solar metal abundances.
Our result is $[\mathrm{Fe} / \mathrm{H}]=-0.56 \pm 0.11$, which agrees very well with the metal abundance of components $\mathrm{A}$ and $\mathrm{B}$ and also with the result from Anglada-Escudé et al. (2013, who used the same method). The value reported by Neves et al. (2014) is $[\mathrm{Fe} / \mathrm{H}]=-0.5 \pm 0.09$, which is also consistent with the system's higher mass components.

We conclude from this first test that the new PHOENIX ACES models provide reliable results in $\log g$ and $T_{\text {eff }}$. For metallicity, the situation is more difficult. Our models indicate higher metallicities than other methods in the three stars for which temperature and gravity are constrained from knowledge about the stellar radii. In the fourth object, which has information on metal abundance from its higher mass companions, our methods provide results that are consistent with other methods. Further investigation including comparison of metallicities in 

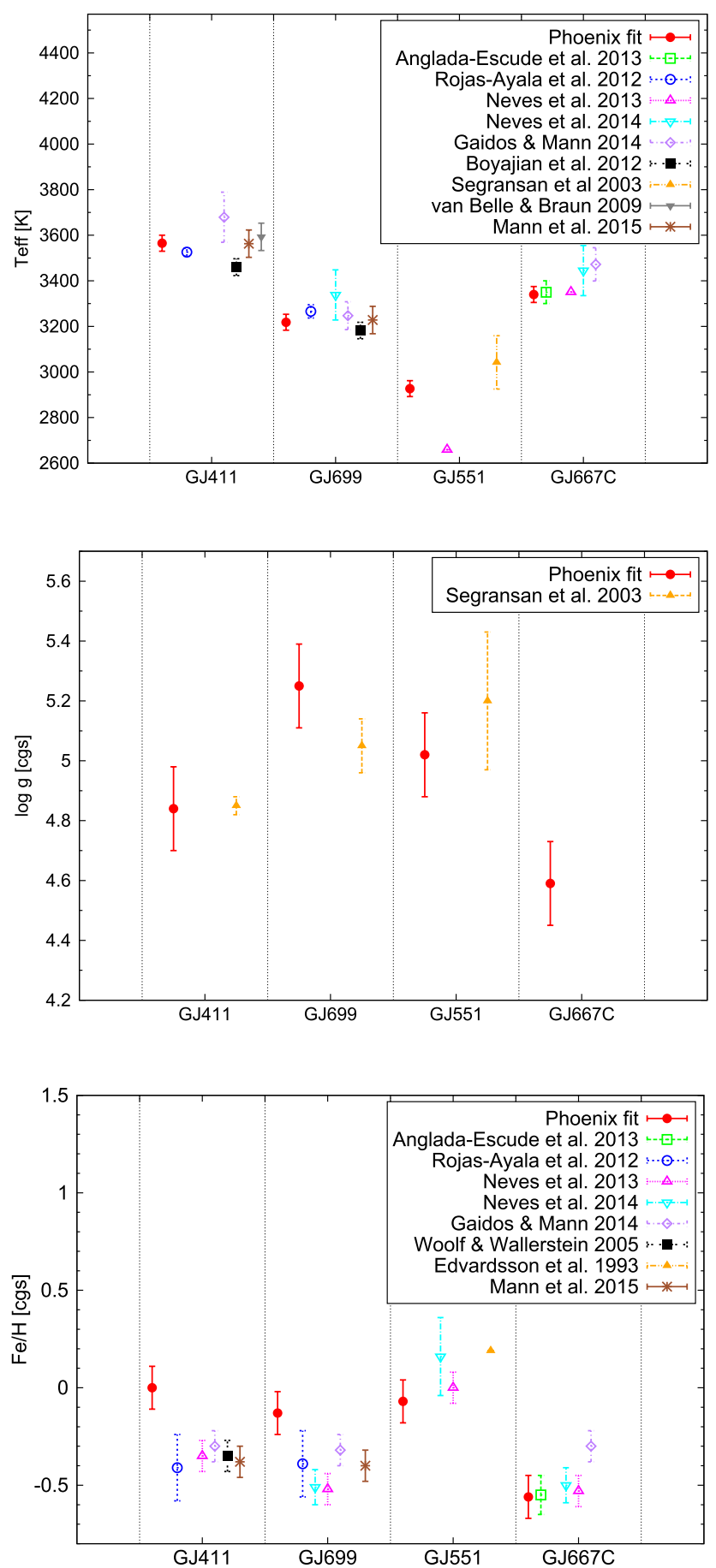

Fig. 6. Comparison between our Phoenix results and results reported in the literature values. Top panel: effective temperature, middle panel: surface gravity, bottom panel: metallicity.

other binary systems will be carried out in a subsequent paper when a larger M-dwarf sample will be investigated.

\section{Summary and discussion}

We have determined atmospheric parameters from highresolution optical spectroscopy in a few benchmark M dwarfs with the new PHOENIX ACES models. The models include an improved description of physical processes in low-temperature atmospheres and are expected to provide a better fit to spectroscopic observations and accurate atmospheric parameters.
We identified useful spectroscopic indicators with which the three parameters can be constrained within small statistical uncertainties. Uncertainties due to degeneracies between spectra with different parameters are $\sigma_{T_{\text {eff }}}=35 \mathrm{~K}, \sigma_{\log g}=0.14$, and $\sigma_{[\mathrm{Fe} / \mathrm{H}]}=0.11$.

The main purpose of the paper was a comparison of the model spectra to stars with independent information on atmospheric parameters. Temperature, surface gravity, and metallicity were determined for four stars. For three of the four, we compared our model results to temperature and gravity known from interferometric radius measurements, luminosity, and parallax. In all six parameters, the agreement is excellent (within $1 \sigma$ ). Metallicity measurements are also available for the three stars, but they are inconsistent with our results. On average, our model fits are 0.31 dex higher than literature values, which is similar to the offset found by Johnson \& Apps (2009). A comparison with results in the NIR (Rojas-Ayala et al. 2012) showed an average deviation of 0.33 dex for GJ411 and GJ699. The same difference was found when comparing our results for these two stars to Mann et al. (2015). At this point, we cannot clearly identify the reason for this discrepancy. One possible explanation is that our improved models provide a better description of the cool atmospheres and therefore more accurate metallicities than other methods. However, we cannot exclude the possibility that our metallicities systematically overestimate the metal abundances.

Rajpurohit et al. (2014) determined stellar parameters of M dwarfs using BT-Settl models. They reported good fitting of the TiO-bands, but rather poor fitting of the $\mathrm{K}$ - and $\mathrm{Na}$-line pairs for later-M dwarfs, with the observed lines being broader and shallower than the models. We observe a similar behavior (see spectra for GJ 551 and GJ 699 in Fig. 5), although in our case the models show broader lines. However, this might be an indication that current models still have problems to reproduce lines in the lower temperature and maybe lower metallicity range.

Pavlenko et al. (2015) investigated the binary system G 224$58 \mathrm{AB}$, consisting of a cool $\mathrm{M}$ extreme subdwarf and a brighter $\mathrm{K}$ companion. They determined abundances of different elements for the bright component using synthetic spectra calculated with the WITA6 program (Pavlenko 1997) and checking the agreement to the spectrum with models with different parameters. Abundances for the cooler $\mathrm{M}$ companion were determined independently by using NextGen and BT-Settl models (Hauschildt et al. 1999). No comparison with literature values was made, therefore the validity of the abundances cannot be verified. However, they proved the binarity of the system by showing that both components have the same metallicities derived by different methods. This shows that even previous-generation models were capable of reproducing metallicities using specific lines, which indicates that the new-generation models might work even better using whole molecular bands.

To address our offset in metallicity, we investigated a fourth star that is a member of a multiple system with known metallicities. In this case, our result agrees with the literature values reported for the more massive components, in which determinations of metallicity are better established. While this comparison supports the interpretation that the new models accurately describe cool atmospheres, it does not solve the puzzle of why our metallicities disagree with other M-dwarf metallicity scales because in this particular star other determinations (are designed to) agree as well.

High-resolution spectra of low-mass stars can potentially be used to determine atmospheric parameters and even individual element abundances to high accuracy. We have shown that the new PHOENIX grid provides a good set of models to start such 
V. M. Passegger et al.: Fundamental M-dwarf parameters from high-resolution spectra using PHOENIX ACES models. I.

a comparison. We plan to use our method to derive atmospheric parameters from many M-dwarf spectra, and this analysis should also include a comparison to other metallicity scales. If the real metallicities of $M$ dwarfs are indeed several tenths of a dex higher than currently assumed, this would have serious ramifications for our understanding of planet formation and the local stellar population. Therefore, a consistent understanding of all spectral features in cool atmospheres is mandatory.

Acknowledgements. We thank Barbara Rojas-Ayala and Andreas Schweitzer for fruitful discussions about low-mass star atmospheres and metallicity scales. V.M.P. acknowledges funding from the GrK-1351 Extrasolar Planets and their Host Stars. SWvB acknowledges DFG funding through the Sonderforschungsbereich 963 Astrophysical Flow Instabilities and Turbulence, and the GrK-1351 Extrasolar Planets and their Host Stars. A.R. acknowledges funding from the DFG as a Heisenberg-Professor under RE-1664/9-2 and support by the European Research Council under the FP7 Starting Grant agreement number 279347.

\section{References}

Allard, F., Hauschildt, P. H., Alexander, D. R., Tamanai, A., \& Schweitzer, A. 2001, ApJ, 556, 357

Allard, F., Homeier, D., \& Freytag, B. 2011, in 16th Cambridge Workshop on Cool Stars, Stellar Systems, and the Sun, eds. C. Johns-Krull, M. K. Browning, \& A. A. West, ASP Conf. Ser., 448, 91

Anglada-Escudé, G., Tuomi, M., Gerlach, E., et al. 2013, A\&A, 556, A126

Bean, J. L., Benedict, G. F., \& Endl, M. 2006a, ApJ, 653, L65

Bean, J. L., Sneden, C., Hauschildt, P. H., Johns-Krull, C. M., \& Benedict, G. F. 2006b, ApJ, 652, 1604

Bonfils, X., Delfosse, X., Udry, S., et al. 2005, A\&A, 442, 635

Boyajian, T. S., von Braun, K., van Belle, G., et al. 2012, ApJ, 757, 112

Casagrande, L., Flynn, C., \& Bessell, M. 2008, MNRAS, 389, 585

Dawson, P. C., \& De Robertis, M. M. 2004, AJ, 127, 2909

del Burgo, C., Helling, C., Martín, E. L., et al. 2013, Mem. Soc. Astron. It., 84, 1084

Delfosse, X., Forveille, T., Ségransan, D., et al. 2000, A\&A, 364, 217

Edvardsson, B., Andersen, J., Gustafsson, B., et al. 1993, A\&A, 275, 101

Fischer, D. A., \& Valenti, J. 2005, ApJ, 622, 1102
Gaidos, E., \& Mann, A. W. 2014, ApJ, 791, 54

Gizis, J. E. 1997, AJ, 113, 806

Hauschildt, P. H., Baron, E., \& Allard, F. 1997, ApJ, 483, 390

Hauschildt, P. H., Allard, F., \& Baron, E. 1999, ApJ, 512, 377

Husser, T.-O., Wende-von Berg, S., Dreizler, S., et al. 2013, A\&A, 553, A6

Johnson, J. A., \& Apps, K. 2009, ApJ, 699, 933

Jones, H. R. A., Longmore, A. J., Allard, F., \& Hauschildt, P. H. 1996, MNRAS, 280, 77

Maldonado, J., Affer, L., Micela, G., et al. 2015, A\&A, 577, A132

Mann, A. W., Brewer, J. M., Gaidos, E., Lépine, S., \& Hilton, E. J. 2013, AJ, 145,52

Mann, A. W., Deacon, N. R., Gaidos, E., et al. 2014, AJ, 147, 160

Mann, A. W., Feiden, G. A., Gaidos, E., Boyajian, T., \& von Braun, K. 2015, ApJ, 804, 64

Marsakov, V. A., \& Shevelev, Y. G. 1988, Bull. Inf. CDS, 35, 129

Mould, J. R. 1976, ApJ, 210, 402

Neves, V., Bonfils, X., Santos, N. C., et al. 2012, A\&A, 538, A25

Neves, V., Bonfils, X., Santos, N. C., et al. 2013, A\&A, 551, A36

Neves, V., Bonfils, X., Santos, N. C., et al. 2014, A\&A, 568, A121

Pavlenko, Y. V. 1997, Ap\&SS, 253, 43

Pavlenko, Y. V., Zhang, Z. H., Gálvez-Ortiz, M. C., Kushniruk, I. O., \& Jones, H. R. A. 2015, A\&A, 582, A92

Perrin, M.-N., Cayrel de Strobel, G., \& Dennefeld, M. 1988, A\&A, 191, 237

Rajpurohit, A. S., Reylé, C., Allard, F., et al. 2013, A\&A, 556, A15

Rajpurohit, A. S., Reylé, C., Allard, F., et al. 2014, A\&A, 564, A90

Reiners, A. 2005, Astron. Nachr., 326, 930

Reiners, A., Joshi, N., \& Goldman, B. 2012, AJ, 143, 93

Rice, E. L., Oppenheimer, R., Zimmerman, N., Roberts, L. C., \& Hinkley, S. 2015, PASP, 127, 479

Rojas-Ayala, B., Covey, K. R., Muirhead, P. S., \& Lloyd, J. P. 2012, ApJ, 748, 93

Santos, N. C., Israelian, G., Mayor, M., et al. 2005, A\&A, 437, 1127

Schlaufman, K. C., \& Laughlin, G. 2010, A\&A, 519, A105

Ségransan, D., Kervella, P., Forveille, T., \& Queloz, D. 2003, A\&A, 397, L5

Taylor, B. J. 2005, ApJS, 161, 444

Thevenin, F. 1998, VizieR Online Data Catalog: III/193

Valenti, J. A., Piskunov, N., \& Johns-Krull, C. M. 1998, ApJ, 498, 851

van Belle, G. T., \& von Braun, K. 2009, ApJ, 694, 1085

Wende, S., Reiners, A., \& Ludwig, H.-G. 2009, A\&A, 508, 1429

Woolf, V. M., \& Wallerstein, G. 2004, MNRAS, 350, 575

Woolf, V. M., \& Wallerstein, G. 2005, MNRAS, 356, 963

Zboril, M., \& Byrne, P. B. 1998, MNRAS, 299, 753 